\title{
TOWARDS AN ONTOLOGY MAPPING PROCESS FOR BUSINESS PROCESS COMPOSITION
}

\author{
Célia Talma Martins ${ }^{1,2}$, Américo Azevedo ${ }^{3,4}$, H. Sofia Pinto ${ }^{5}$, Eugénio Oliveira ${ }^{1,3}$ \\ ${ }^{1}$ LIACC-NIAD\&R, Faculty of Engineering, University of Porto \\ R. Dr. Roberto Frias, 4200-465 Porto, Portugal \\ ${ }^{2}$ ISCAP, Rua Jaime Lopes Amorim, s/n, 4465-004 S. Mamede de Infesta \\ ${ }^{3}$ Faculty of Engineering, University of Porto \\ ${ }^{4}$ INESC Porto, Portugal \\ ${ }^{5}$ INESC-ID/IST, Rua ALves Redol, 9, 1000-029 Lisboa \\ talma@fe.up.pt; ala@fe.up.pt; sofia@inesc-id.pt; $\underline{\text { eco@fe.up.pt }}$
}

\begin{abstract}
Business Processes Composition in dynamic and semantic heterogeneous environments as Electronic Institution or Virtual Breeding Environment it's an actual research topic.

We propose a solution based on ontology mapping to solve the semantic heterogeneity problem that the composition of business processes raises in these environments.
\end{abstract}

\section{INTRODUCTION}

Business Processes Composition in dynamic and semantic heterogeneous environments as Electronic Institutions or Virtual Breeding Environments it is an actual research topic. The Semantic Heterogeneity Problem occurs in business processes composition in such semantic heterogeneous environments as it is the case of the Electronic Institution (EI) or a Virtual Organizations Breeding Environment (VBE), which is a community of enterprises that maintain a set of social relations, based on trust, having some common strategic goals. A further level of cooperation and collaboration happens when enterprises belonging to this VBE, take advantage of this situation to catch up business opportunities by engaging temporarily in a so called Virtual Enterprise (VE). The design of InterOrganizational Business Processes (IOBP) in a VBE it is the main concern of our research. As enterprises have different ontologies and languages to define their own business processes this raises the semantic heterogeneity problem.

In order to solve this problem, we will apply an ontology mapping service included in a multi-agent system platform facilitating B2B interoperability during a VE lifecycle (Malucelli, 2006).

Section 2 presents an overview of business processes in Breeding Environments, section 3 presents the semantic heterogeneity problem and section 4 presents our solution to the problem and the state-of-the-art in the field. Finally section 5 presents some conclusion and further research work. 


\section{BUSINESS PROCESSES in BREEDING ENVIRONMENT}

\subsection{Business Processes overview}

Nowadays enterprises achieve their goals through their business processes, e.g. they are process-oriented. Processes are the very heart of every organization because they are the means through which companies create value for their customers. A process-based organization always starts with the identification of the key processes of the company (Vanhaberbke and Torremans, 1999).There are several definitions of business processes such as: "is a structured, measured set of activities designed to produce a specific output for a particular customer of market"(Davenport, 1993) or "...a business process as a collection of activities that takes one or more kinds of input and creates an output that is of value to the customer" (Hammer and Champy, 1993).

We see a business process as a collection of sub-processes (activities) that have one or more inputs, objectives, and one or more outputs.

Process-oriented enterprises have their key business processes identified so they can announce them in the VBE in order to participate in a future VE formation/creation.

These days enterprises are starting to cooperate with other in VE. The lifecycle of these VE has four phases: creation, operation, evolution and dissolution.

\subsection{Business Processes Composition}

We think that business process composition is very similar to semantic web services composition. Like web services, business processes have an input, and output and a transformation function. However business processes are more complex, because they also have objectives, a process name, clients, costumers, actors and the process flow itself. Nevertheless, in it's most basic form, a business process has one or more inputs, objectives and one or more outputs.

Although there are many web services repositories available on the web, sometimes just one web service is not enough to do a functionality required by a user or a software agent. So there should be a way to combine existing services together in order to fulfill the request. This trend has triggered a considerable number of research efforts on web services composition both in academia and industry. Similarly the same happens with business processes: sometimes one business process alone cannot fulfill a business opportunity by itself, so we need to combine in some way existing business processes of the VBE in order to fulfill a client's business opportunity request, thus forming a new VE for that particular business opportunity. There is the need to define a methodology to chain the different business processes of the different enterprises into an interorganizational business process that satisfies a particular Business Opportunity. This is our main research issue: how to achieve the right sequence of the different enterprise's business processes in order to fulfill that particular Business Opportunity.

Based on the definition of the generic IOBP we will make the selection of business processes that satisfy that particular business opportunity. For this selection we will search in the VBE enterprises for the business processes and sub-business processes that are previously defined in the generic IOBP. In the end we will get a final VE as we can see in Figure 1, which responds to our business opportunity and a composition of business processes that will form the IOBP that responds to the business opportunity. The search for business processes can involve a basic negotiation if more than one process is found that satisfies the process we are looking for. In this case, we will select the one that has the lowest price. 


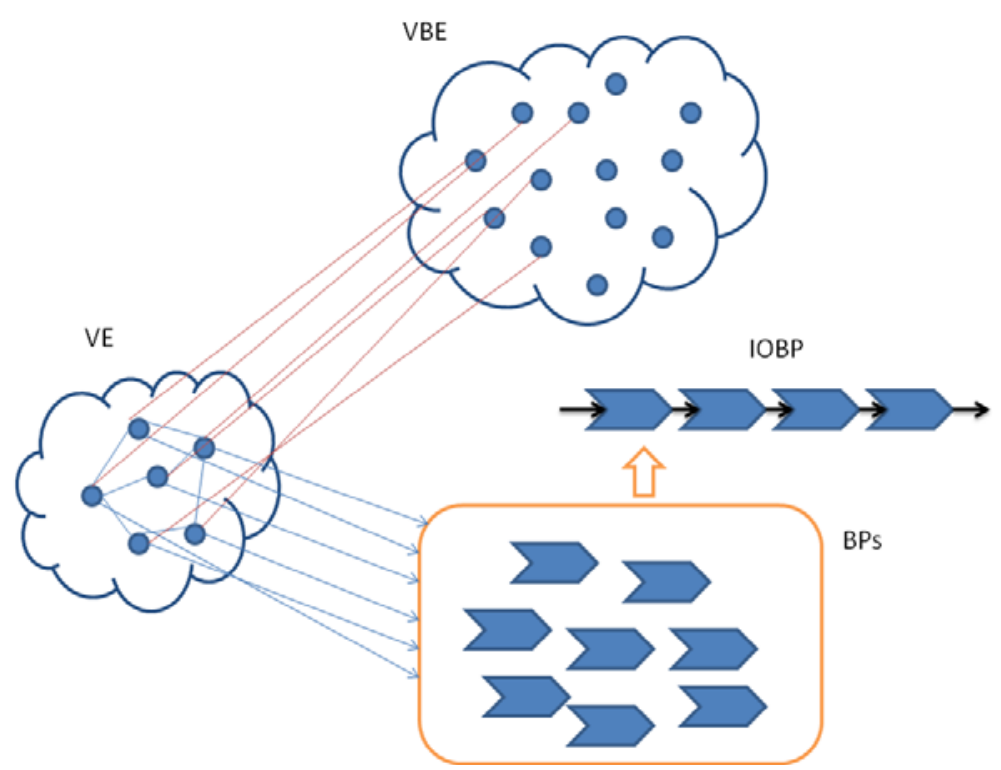

Figure 1. Composition of Business Processes

If the enterprises that belong to the VBE agree on a common ontology there are no misunderstanding problems while searching of similar business processes. However, the enterprises that belong to the VBE usually use different ontologies to represent their own business processes, so the semantic heterogeneity problem will occur.

\section{SEMANTIC HETEROGENEITY PROBLEM}

\subsection{Ontology Mapping}

Ontologies were developed in order to facilitate knowledge sharing and reuse. An ontology is an explicit formal specification of the concepts in the domain and the relations among them (Gruber, 1993). An ontology defines a common vocabulary for researchers who need to share information in a domain.

Ontology mapping is the process of finding correspondences between concepts represented in different ontologies. If two concepts correspond they should mean the same or closely related things (Dout et al, 2003). Ontology mapping uses similarity measures to see if two terms representing a concept, are either equal or mean exactly the same or have a strong similarity or are not similar at all. The same applies to both relations and properties as well as to all the entities included in the ontologies. Mapping is important once different enterprise software agents using different ontologies have to exchange and possibly combine their own business processes. There is thus a need for comparing if two business processes are equal, mean exactly the same, have a strong similarity or are not similar at all. 
A business process has a name, inputs and outputs, and the name, inputs and outputs are composed of concepts that need to be compared. We have some problems that may occur:

- two business processes may have the same name but different inputs and outputs and so have completely different meanings

- two business processes may have the same name, equal inputs and outputs and so are exactly the same business process

- two business processes may have different names but have equal inputs and outputs and so are exactly the same business process

- two business processes may have different names, inputs and outputs and so have no similarity

So Ontology Mapping is a possible solution to resolve the semantic heterogeneity problem.

\subsection{Ontology and Business Process Management - state-of-the-art}

A method for agents to develop local consensus ontologies is proposed in (Williams et al., 2003) to aid in the communication in a business-to-business multi-agent system. They also compare variations of syntactic and semantic similarity matching to form local consensus ontologies with and without the use of a lexical database. This approach allows to find syntactic and semantic similarity by comparing two ontologies at a time with each other without the use of a global common ontology. It then merges these ontologies into a local, consensus ontology. If one agent determines that another agent's concept is similar, or equivalent, to its own concept either syntactically or semantically, it can add this concept and its associated relations to its local consensus ontology.

An approach for (semi)-automatic detection of synonyms and homonyms of process element names is presented in (Ehrig et al., 2007) in order to support semantic process model interconnectivity and interoperability by measuring the similarity between business process models semantically modelled with the Web Ontology Language (OWL). The authors further show in this paper, that by using the three similarity measures, syntactic, linguistic and structural, they can compute similarity degrees between a pair of process element names and between a pair of process models. The syntactic similarity degree is computed by comparing the number of common characters in the element names (e.g., confirmation vs verification). In order to measure similarity between two strings, the (Levenshtein) edit distance method is used. The linguistic similarity degree relies on a dictionary to determine synonyms. In this case, WordNet was used. However syntactic and linguistic similarity measures by themselves do not exploit the context of the names. That is done with structural similarity measures, which helps to detect primarily homonyms.

In (Malucelli et al., 2005) it is combined the use of ontologies and agent technologies to help in solving the semantic heterogeneity problem in e-commerce negotiations. Thereby, the focus is on ontologies, whose specifications include a concept (item/product), its characteristics (attributes) with the correspondent data types, a natural language description explaining the meaning of the concept, and a set of relationships between these concepts. This approach aims at creating a methodology that assesses a lexical and semantic similarity among concepts represented in different ontologies without the need to build a priori a shared ontology. The lexical measures are used to compare attributes and relations between concepts.

The authors in (Mtatskin et al, 2005 ) describe their own method for Web Services (WS) selection and composition based on Linear Logic. According to the authors the 
complexity of selecting and composing web services arises from two sources: it is not always easy to define selection criteria for a WS; WS can be developed by different organizations, which provide different offers, so, the ability of efficient integration of possibly heterogeneous services on the web becomes a complex problem. Their approach to WS Composition is based on a logic and marketplace system architecture that supports agent communication, negotiation and semantic reasoning. The authors claim that the use of marketplaces provides a support for composition of services based both on flow models and AI planning. The innovation of the presented work lays on the novelty that networks of marketplaces may allow to specify more flexible WS process and data flows with decentralized control than traditional workflow models. The composition of services is based on a functional specification, without taking into account low level details, such as operational environment or communication protocol.

The author's in (Berardi and Giacomo, 2005) envision an advanced Web Service registry providing support for semantic discovery, i.e., where the WS search is done by considering user specification involving WS capabilities and behaviour. The fundamental idea is to enable organizations to seamlessly compose BPs and dynamically integrate them with the partners' processes, by means of lightweight workflow-like technologies. Therefore, the WS composition framework will form a conceptual basis to define how internal BPs can be dynamically integrated with those of other organizations as valueadded WS.

In (Paolucci et al, 2002) The authors claims that the location of web services should be based on the semantic match between a declarative description of the service being sought, and a description of the service being offered. An automatic matching algorithm is proposed for the automatic dynamic discovery, selection and interoperation of web services. DAML-S was adopted as the service description language because it provides a semantical view of web services.

In (Gómez-Peres et al, 2004) it is proposed a framework for design and (semi) automatic composition of semantic web services at a language-independent and knowledge level. This framework is based on a stack of ontologies that (1) describe the different parts of a SWS; and (2) contain a set of axioms that are really design rules to be verified by the ontology instances. Based on these ontologies, design and composition of SWS can be viewed as the correct instantiation of the ontologies themselves.

\subsection{Proposed Solution}

Our work is inserted in the configuration phase which precedes the operation phase.

We will focus on the definition of the Inter-Organizational Business Processes for a particular Business Opportunity, and for that we must select and choose the right business processes of the enterprises that belong to the breeding environment in order to fulfill that Business Opportunity.

We are assuming that a first iteration of the VE formation process has already been achieved (Macedo, 2001), e.g., we have a first set of enterprises that have been selected based on criteria such as their specific competencies and prices for a particular Business Opportunity (BO).

Following that preliminary stage there is the need to identify all the business requirements which are important for the definition of the generic IOBPs (involving all the selected partners) which will be considered as an input in the problem to be solved. This will be done by the management people, because this a decision support system that helps the management people take a decision, e.g., the main idea is to know if we can articulate the business processes of the different enterprises of the VBE to respond to that 
BO. The enterprise management people designs the IOBP that will satisfy a particular business opportunity, and this IOBP is an input of our problem.

The enterprises announce their competencies, skills, resources and public business processes in this dynamic heterogeneous environment. However, it's not probable that they all have the same ontology. So when a business opportunity arises, the management people define a generic inter-organizational business process (IOBP) which is an input of our problem. Based on this IOBP we will select the most appropriate sub-business processes of the IOBP in the VBE that satisfy the IOBP. But, as already mentioned the different business processes of the different enterprises do not have a common ontology, so this raises the so called Semantic Heterogeneity Problem.

We have two degrees of complexity in our problem. First we will assume that all the enterprises have the same ontology to define their public business processes.

Let us present an example:
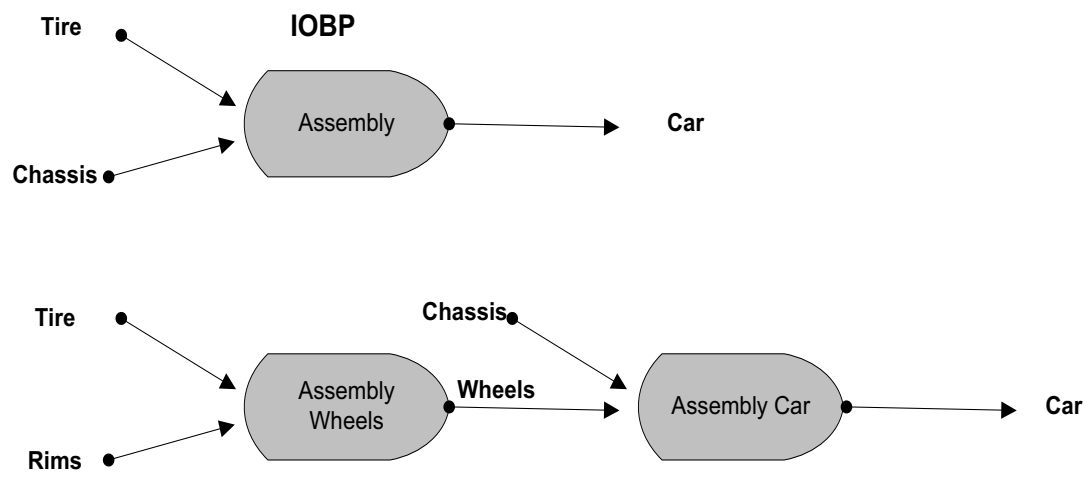

Figure 2. Business Process Composition - same ontology

In Figure 2 we can see that for we respond to the IOBP we have to gather the two upper sub-business processes: Assembly Wheels and Assembly Car, because the two business processes together have the same inputs and outputs than the generic IOBP. We are supposing that Assembly Wheels and Assembly Car are processes from different enterprises. We can respond to the business opportunity because we can gather two business processes from two different enterprises that satisfy the business opportunity. As we already mentioned, this will be done through the development of an algorithm that composes sub-business processes based on some constraints. For example one of this constraint-based rules indicates that I may only compose two business processes if the output of the first is the input of the second.

Now suppose that the enterprises have different ontologies and so implying that their business processes are differently described. The same example above would be described as follows in figure 3 : 

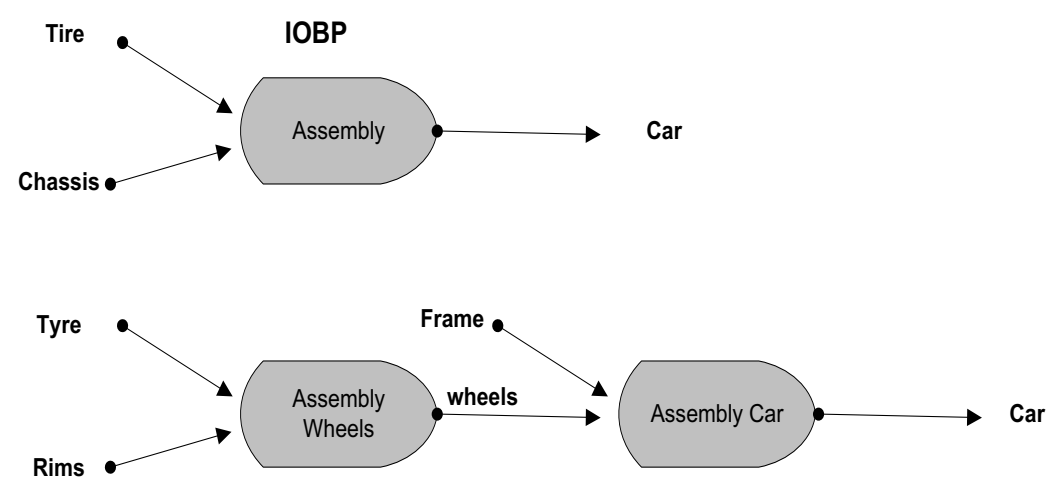

Figure 3. Business Process Composition - different ontologies

How should the system know that Tire means the same that Tyre and that Frame means the same as chassis? This is a more complex problem, which we call semantic heterogeneity problem. How can one ensure that the processes from the different enterprises have the same understanding regarding the issues that are subject to the composition of business processes?

And in order to solve this problem, we think that the use of ontology mapping will help us dealing with this problem. We must have a mapping algorithm between the two ontologies that tell us that Tire means the same as Tyre and that Chassis means the same as Frame.

\section{CONCLUSIONS AND FURTHER RESEARCH}

We think that the use of ontologies, more precisely ontology mapping, will help us resolving the semantic heterogeneity problem, in order to compose the right business processes that are defined through different ontologies.

To see if two business processes are similar we will use an ontology matching algorithm that is based on a previous work (Malucelli, 2006). This work uses semantic similarity measures to compare if two terms are equals.

We need to develop an algorithm that will lead us to a soundness composition of business processes considering their inputs, outputs and objectives.

Another interesting issue would be to learn from previous compositions. 


\section{REFERENCES}

1. Malucelli A. Ontology - based Services for Agents Interoperability, Phd Thesis, Faculdade de Engenharia, Universidade do Porto, 2006.

2. Vanhaverbeke, W., Torremans, H. Organizational structure in process-based organizations", Knowledge and Process Management 1999; Vol. 6 No.1: 41-52.

3. Davenport, Thomas. Process Innovation: Reengineering work through information technology, Harvard Business School Press, Boston, 1993.

4. Hammer, Michael and Champy, James, Reengineering the Corporation: A Manifesto for Business Revolution, Harper Business, 1993.

5. Gruber, T.R. A Translation Approach to Portable Ontology Specification. Knowledge Acquisition 1993; 5: 199-220.

6. Dou D., Mcdermott D., Qi P., Ontology Translation on the Semantic Web. In Proceedings of International Conference on Ontologies, Databases and Applications of Semantics (ODBASE 2003). LNCS 2888, Springer-Verlag. Berlim Heidelberg, pp. 952-969,2003.

7. Andrew Williams, Anand Padmanabhan, M. Brian Blake, Local consensus ontologies for B2B-oriented service composition, Proceedings of the second international joint conference on Autonomous agents and multiagent systems, July 14-18, 2003, Melbourne, Australia, 2003.

8. Marc Ehrig, Agnes Koschmider, Andreas Oberweis, Measuring Similarity Between Semantic Business Process Models, in Proceedings of the 4th Asia-Pacific Conference on Conceptual Modelling (APCCM 2007), Ballarat, Victoria, Australia, 2007.

9. Andreia Malucelli, Daniel Palzer, Eugénio Oliveira, Combining Ontologies and Agents to help in solving the Heterogeneity Problem in E-Commerce Applications, in International Workshop on Data Engineering Issues in E-Commerce (DEEC 2005), IEEE Computer Society. pp. 26-35. Tokyo, Japan. April 2005.

10. M. Matskin, P. Küngas, J. Rao, J. Sampson, S. A. Petersen. Enabling Web Services Composition with Software Agents. In Proceedings of the Ninth IASTED Int. Conference on Internet and Multimedia Systems and Applications, IMSA 2005, Honolulu, Hawaii, USA, August 15-17, 2005, ACTA Press, pp. 93-98, 2005.

11. Berardi D., Giacomo G., Automatic Composition of Process-based Web Services: a challenge, in Second European Conference on Semantic Web, May 10-14, Chiba - Japan, 2005

12. Paolucci, M., Kawamura, T., Payne, T. R. and Sycara, K. Semantic Matching of Web Services Capabilities. In: International Semantic Web Conference (ISWC), 9 - 12 June, Sardinia, Italy. 2002.

13. Asunción Gómez-Pérez, Rafael González-Cabero, and Manuel Lama. A Framework for Description, Composition, and Evaluation of Semantic Web Services. IEEE Intelligent Systems. Special Issue on Semantic Web Services. 2004

14. Macedo A.P., Metodologias de Negociação em Sistemas Multi-Agentes para Empresas Virtuais. Tese de Doutoramento. Faculdade de Engenharia, Universidade do Porto, 2001. 